\title{
Secure E-learning using the Holistic and Immune Security Framework
}

\author{
Jeffy Mwakalinga, Stewart Kowalski and Louise Yngström \\ Department of Computer and Systems Sciences, \\ Stockholm University / Royal Institute of Technology, Sweden \\ jeffy@dsv.su.se, stewart@dsv.su.se, louise@dsv.su.se
}

\begin{abstract}
This paper describes how to secure e-learning systems by applying the Holistic and Immune Security Framework. E-learning has great potential for developing communities but security of e-learning systems has not been fully addressed. We have developed a security framework that considers culture of users and environments where information systems operate. We apply the holistic approach to secure elearning systems. The holistic and immune security framework is a function of the deterrence, prevention, detection, response, and recovery system. The security framework makes an E-learning system learn to adapt to environments and to culture of users. We apply the principles of immune system to secure E-learning systems. We describe how to secure the weak links that are created by culture of users in E-leaning systems.
\end{abstract}

\section{INTRODUCTION}

E-learning is a special form of learning in which material is delivered electronically to learners in different cultures and environments [6]. We have assumed that users in E-learning systems is just another component which must follow instructions as other technological components, which has resulted in people serving technology and not technology serving people. Culture affects the way users interact with different information systems [14]. Elearning systems should learn to adapt to environments where they operate and to cultures of users. An e-learning environment consists of five layers as shown in Figure 60. The first layer is users' gate. In this gate, different users like owners, designers, developers, teachers, authors, reviewers, learners, and administrators are identified and given access the e-learning system. The second layer contains the common management services like user management, collaboration management, and courses' catalog management. The third layer has e-learning services and consists of learning content management system (LCMS) [20], learning management system (LMS), assessment, evaluation, business operations, and administration. The learning content management system is concerned with content development in which authors, reviewers, content experts, administrators submit, review edit contents. [20]. Learning management services are concerned with content delivery services and interaction services with learners. The fourth layer consists of databases for all the information of the e-learning environment. The fifth layer consists of network infrastructure. This layer has delivery networks for audio, voice of IP, video, IP data, servers, and protocols like FTP, HTTP, SOAP, XML, and TCP/IP as shown in Figure 60.

\section{HOLISTIC AND IMMUNE SECURITY FRAMEWORK}

We have created a holistic and immune security framework as shown in Figure 61. The security framework bases on the SystemicHolistic Approach [11] and the principles of the immune system [8]. In the security framework, we apply the holistic approach to 
secure information systems. We believe that there is much more to information security than technology and communication protocols. Therefore, we also consider different factors that affect information security like environments, culture, laws, and ethics of users, economy, and other issues. The security framework is a function of value-based chain functions [2]. The value-based chain for information security was developed by [3]. The security value-based chain contains deterrence, prevention, detection, response, and recovery functions. The security framework consists of the following components: the management system, the adaptability system, the deterrence sub system, the prevention sub system, the detection sub system, the response sub system, and the recovery sub system. The management system contains the agent generator, databases, integrated security system, special analyzer, system manager, security management, and fault tolerance management. The agent generator creates agents that provide security services to the different components of the security framework and the e-learning system. The integrated security system manages certificates, smart systems, authorization systems, and database systems.

We apply the special analyzer to study all the inputs that are new to the different components of the security framework and the e-learning system. The system manager takes care of administration of the security framework. Security management takes care of risk management, policy management, compliance management, and business continuity management in the E-learning system. The fault tolerance manager is responsible for detecting errors, assessing damages, confining damages, treating faults, locating faults in the security framework and the in the e-learning system [6]. The adaptability system provides measures for making an e-learning security system learn to adapt to environments. A deterrence sub system scares away attackers of an e-learning system. Prevention sub system guides the territory of an e-learning system and its entities. Detection sub system detects attacks and abnormalities in an e-learning system. Response sub system responds to attacks and intrusions in an e-learning system. Recovery sub system restores an attacked elearning system back to normal. In every sub system, we analyze, control and process all the inputs and then take out poison in the inputs by applying the feedback systems from cybernetics [11] as shown in Figure 61.

\subsection{Provision of SECURITY IN AN E- LEARNING SYSTEM}

The holistic an immune security framework methodology that we apply for securing an elearning system is as follows [21]. We start by analyzing the threat agent to the e-learning systems based on the socio-technical economical model. Then we classify the assets of the e-learning system and perform risk management. The next step is to analyze environments where the e-learning system is operating. Thereafter we assess the effects of culture, laws, ethics, and other social issues of e-learning system's users to the information security of the system. In the next step, we apply socio-technical measures [19] to secure the weak links that have been created by culture of e-leaning system's users. Then we provide measures for making an e-learning security system learn to adapt to environments and culture of users.

The next step is to analyze how to distribute economical resources to the deterrence, prevention, detection, response, and recovery security functions of an e-learning system. Then we educate users electronically on social engineering and other security issues. In the last step we evaluate the outcomes of the implementation of the holistic and immune security framework based on the plan, do, check, and act process for continuous security improvement outlined in ISO27001 [22]. 
First layer: Users' gate (owners, designers, developers, teachers, authors, content experts, learners, and administrators)

Second layer: common management services (user management, collaboration, courses' catalog management)

Third layer: e-learning services (learning content management services, learning management services, assessment, evaluation, business operations, administration)

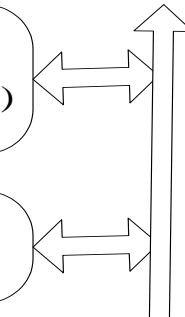

Fourth layer: databases

Fifth layer: network infrastructure (delivery networks, audio, voice of IP, video, FTP, HTTP, SMTP, SOAP, XML, TCP/IP servers, etc)

Holistic and immune security framework

FIGURE 60: LAYERS OF AN E-LEARNING SYSTEM AND THE HOLISTIC IMMUNE SECURITY FRAMEWORK
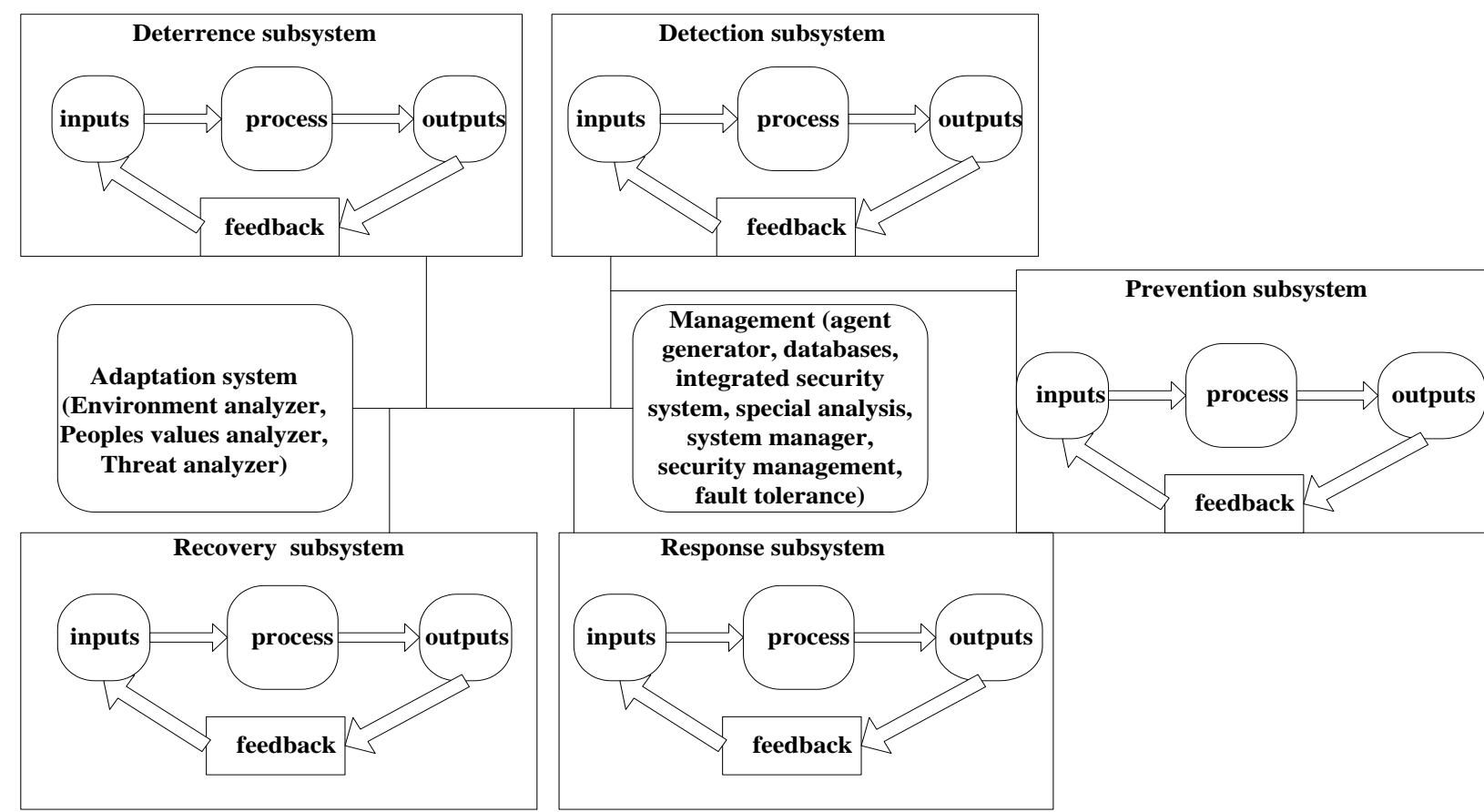

FIGURE 61: THE MODEL OF THE HOLISTIC AND IMMUNE SECURITY FRAMEWORK 
All the components of an e-learning system users' gate, common management services, elearning services, databases, and network infrastructures are given identities and means to authenticate them. These components are then registered in the database of the security framework. We secure e-learning system's information when it is being processed, stored in databases, in transmission, when collecting information, and when displaying, in all components of the e-learning system.

The most common scenarios in e-learning include teacher centered, evaluation centered, and collaboration centered [4].

In teacher centered scenarios a teacher has to provide material, monitor students, assess students, learn about students, and interact with learning environments, collaborate with other teachers in cases where different modules are integrated. Risks in this scenario include bogus material could be loaded to course websites. Students could gain access to teachers' login credentials. Course material could be changed by unauthorized people; course web sites could be attacked [5]. The software agents provide authentication, authorization, non-repudiation, availability, integrity, privacy, anonymity, and confidentiality security services. Teachers should be identified, authenticated, and authorized before interacting with e-learning environments. The software agents provide privacy and anonymity for teachers. There are some cases where identities of teachers and elearners are necessary to remain classified. This is especially applied when teachers and e-learners are working in intelligence, military and other government ministries where it is necessary to remain classified. In the evaluation cantered scenario, the risks include: people masquerading as students; and students getting outside help in writing tests; submitted answers could be copied or altered by non authorized parties; tests could be accessed beforehand; marks could be changed [5]; and so on. In this scenario, software agents provide authentication, authorization, privacy, integrity, availability, confidentiality, anonymity, and nonrepudiation. The e-learning security system identifies, authenticates, and authorizes e- learners before performing any tasks. Evaluators should also be identified, authenticated and authorized before performing any assessments. Privacy should be provided so that marks, grades and other classified information could remain secret. Integrity is provided at all levels so that assessed and non-assessed information should not be modified. The servers and all systems providing e-learning services should be available all the time. Some e-learners and teachers prefer anonymity so software agents provide anonymity security service. Both students and evaluators sign information. In collaboration-centered scenarios, e-learners work in groups from different locations. The software agents register, monitor, protect, and assess communications among students [4]. In this scenario, the e-learning security system identifies, authenticates, and authorizes elearners before participating in collaboration groups. The software agents provide integrity, confidentiality, non-repudiation, privacy security services in this scenario. Different cultures prefer different mechanisms for implementing security services due to different levels of computer literacy and different cultural values. The Software agents provide multiple authentication schemes, multiple identification schemes, multiple authorization schemes, multiple nonrepudiation schemes, multiple confidentiality schemes, and multiple integrity schemes, multiple anonymity schemes, and multiple privacy schemes. We provide security services using software agents.

\subsubsection{SOFTWARE AGENTS PROVIDE SECURITY SERVICES.}

We apply software agents to provide security services in the different components of an elearning system and the security framework. All components of the security framework request specialized software agents for providing security services in the e-learning system. We apply the principles of the immune system to create software agents. The immune system has B-cells and T-cells. The bone marrow creates B-cells while the thymus generates T-cells [8]. We apply the immune system features, which include multi-layered structure, local detection, diversity, 
autonomy, adaptability, dynamically changing coverage, and identification. The agent creator provides the features of the immune system in the following way [8]. The principle of distribution - B-cells and T-cells detect the presence of infections locally without coordinating with each other. The mobile agents perform tasks in analogy to immune system cells in different subsystems. Every software agent can detect intrusions and abnormalities locally. The multi-layered principle - The immune system applies multiple layers to provide overall immunity in body. The security framework provides multiple protections in e-leaning components. The principle of autonomy - The immune system autonomously classifies and eliminates foreign cells and the immune system repairs itself by replacing damaged cells. This behavior is suitable but its implementation is challenging as technology still is not ready, but we model this partly by having an odd number of agents vote for every decision. The agent creator trains software agents of to make intelligent decisions. We apply the fuzzy logic controllers to train the software agents to make intelligent decisions [7]. Fuzzy logic is a concept in which objects or entities can partially belong to a set. The objects can for instance belong to a certain set by $50 \%$. The range of belonging is $0-100 \%$. In classical sets, either an object or an entity is inside or outside a particular set [7]. We apply the fuzzy logic in detection system decisions because the differences between normal and abnormal behaviors in networks are not distinct but fuzzy [7]. The principle of adaptability - the immune system is able to detect and learn to detect new foreign cells and retains the ability to recognize previously seen foreign cells through immune memory. This feature is not new it in computer systems, though determining that a certain program is malicious with $100 \%$ is a hard problem. We model this by artificial neural networks [8] and genetic programming. The principle of dynamically changing coverage The immune system cannot produce a large enough set of detectors at any moment, so it maintains a random sample of its detectors that circulates throughout the body. This is because there are approximately $10^{16}$ foreign cells and these have to be distinguished from approx. $10^{5}$ 'self'- cells. We model this principle by having one agent detect, prevent or deter multiple intrusions, attacks, abnormalities and viruses. The principle of identification -The immune system marks all the cells belonging to the human body as 'self', considers all other foreign cells are as 'non self'. The mobile software agents recognize normal patterns and regard any other unknown patterns as malicious. We model this principle by providing identities to the all the objects of a system in the form of mini-certificates [9]. We have designed a system of mobile software agents for providing different security services in the sub systems. These include helper and killer agents, authentication agents, confidentiality agents, authorization agents, Non-repudiation and integrity agents, and third-order feedback agents system. We secure the mobile software agents before performing different tasks in the security framework and in the e-learning system as described in [10].

\section{ADAPTABILITY OF THE E- LEARNING SECURITY SYSTEM}

Users of different cultures and in different environments use e-learning systems. An elearning security system has to learn to adapt to the values of users of different cultures and environment where it is operating. In order to provide measures for adaptability we apply different analyzers to study environments, culture of users of e-learning systems. We also study models that adversaries apply to attack e-learning systems.

\subsection{ENVIRONMENTAL ANALYZER}

The environment analyzer provides measures for analyzing environments where an elearning system is running. The analysis bases on the Systemic-Holistic Approach [11], the Cybernetic structural model [1] and the Viable System model [12]. Examples of environments affecting information systems include an operating system, computer hardware, intranet, Internet Service Provider 
(ISP). The adaptability system of the security framework monitors and records the environmental disturbances, the essential variables, and regulatory variables to the elearning system over a period of time [1] as shown in Figure 62. The adaptability system receives environmental disturbances as inputs through the different components of the elearning system and the security framework. The system creates a table of transformations of environmental disturbances, essential variables and regulatory variables. There is a controller of the desired essential variables for the e-learning system and the security framework. This controller mixes the data in the transformations table with the monitored environmental disturbances, essential variables, and regulatory variables to produce the harmless inputs to the e-learning system. The table of transformations represents every possible action that the regulator could apply in response to every environmental disturbance, resulting into a state with the essential variables that must be maintained to keep the e-learning system and the security framework in a stable state. The adaptability system applies the recorded data to create probabilistic models to forecast the future environmental disturbances [9] and thereby foresee how the security framework and the e-learning system would react to those future disturbances. The adaptability system of the security framework is responsible for making sure that the e-learning security system learns to adapt in dynamic environments.

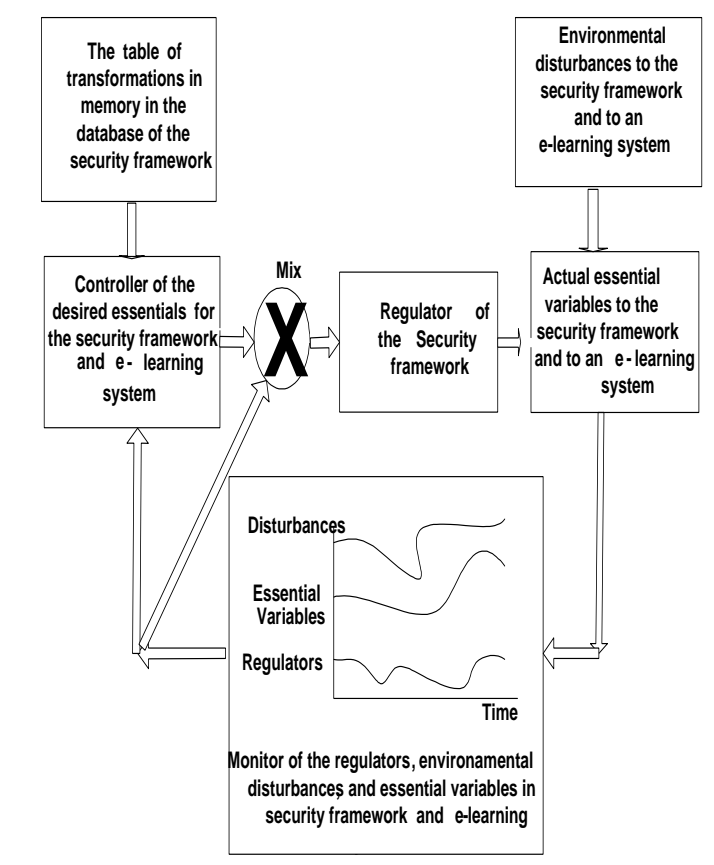

FIGURE 62: THE MODIFIED CYBERNETIC STRUCTURAL MODEL

\subsection{E-LEARNING SYSTEM USERS' CULTURAL VALUES ANALYZER}

There have been concerns about the role of culture in information systems [13]. Culture has been defined as a set of values, attitudes, and behaviors that people learn or are passed over to them over a period of time [14]. There is a general agreement among information system researchers that culture affects the way individuals' interact with complex information systems [13]. This applies to elearning systems as well. There is a need to develop models to measure the effect of culture on individuals when interacting with information systems. Researchers made a survey in three different cultures, Moroccan, Surinamese, and Dutch on user experiences on e-government sites [14]. The conclusion was that people with different culture backgrounds experience different problems in using e-government applications. We could deduct from this conclusion that users with different cultural backgrounds experience different problems in using e-learning systems. The e-learning users' value analyzer provides measures for analyzing how users' culture, traditions, laws and ethic affect elearning security system. We have established an informal cultural model for predicting the 
behavior of e-learning system users of different cultures. The informal cultural model bases on the General Living System [15], Hofstede's Culture Consequences [16] Worldview theory [17], Social Identity theory [18], and computer literacy. This cultural model predicts the behavior and preferences of e-learning system users of different cultures. In some cases, cultural values create vulnerabilities and weak security links in elearning systems. We apply the sociotechnical system [19], Security by Consensus [19], and the Systemic-Holistic Approach [11] to provide socio and technical security controls where culture, traditions, laws, and ethics create a weak link in e-learning security systems. We apply knowledge that we again from the e-learning users' values analyzer to understand, explain, predict and to control. We create policies and procedures to forbid actions that create weak security links in e-learning security system. The results from the e-learning users' values analyzer will show us actions that create vulnerabilities in an e-learning security system. We will assign values to different actions. We forbid actions whose consequences bring negative values.

\subsection{E-LEARNING SYSTEM THREAT ANALYZER}

The threat analyzer deals with understanding the methods, tools and capacity of adversaries, which they apply when attacking e-learning systems. The adversary of elearning systems investigates the tools, methods and processes that an information system is applying to defend in the different subsystems: deterrence, prevention, detection, response and recover. The adversary of elearning systems also finds out how much financial resource e-learning systems allocate in tools, methods and processes for the deterrence, prevention, detection, response, and recovery sub systems. This information helps the adversary of e-learning systems to determine weaknesses in the different sub systems. The information gathered so far will assist the adversary of IT to decide whether it was possible to attack and get out fast without leaving any evidence.

\section{CONCLUSION}

We have described how to protect an elearning system by applying the holistic and immune security framework. We apply the holistic approach to provide secure e-learning system using the principles of the human's immune system. Information security is not just technology and communication protocols and so we consider other factors like environments and values of users of elearning systems. The security framework provides measures that help an e-learning system learn to adapt to environments and to culture of e-learning users. We provide multiple security service schemes to accommodate users with different computer literacy levels and cultural backgrounds. The mobile agents provide security services in an e-learning system. Limitation is that the security framework has not yet been fully implemented. Future work will include fully implementing the security framework and to measure performance of the framework.

\section{REFERENCES}

[1] R. Ashby, Introduction to Cybernetics, Chapman \& Hall, London, 1956.

[2] M.E. Porter, Competitive Advantage, the Free Press, New York, 1985.

[3] S. Kowalski, and N. Edwards, a security and trust framework for a Wireless World: A Cross Issue Approach, Wireless World Research Forum no. 12, Toronto, Canada, 2004

[4] G.C. Webber, M.F Lima, M.E. Casa, A.M. Ribeori, Towards Secure e-learning applications, Journal of software, Academy publisher, Oulu, Finland, pp 60-68.

[5] E. Kritzinger, and S.H. von Solms, E-learning: Incorporating Information Security Governance, Issues in Informing Science and Information Technology, Ipswich USA, pp 319-325.

[6] P.A. Lee, and T Anderson, Fault Tolerance Principles and Practice. Springer-Verlag, Newcastle, 1990.

[7] D. Dasgupta, J. Gomez, F. Gonzales, M. Kaniganti, K. Yallapu, and R. Yarramsettii, "MMDS: Multilevel Monitoring and Detection System", Intelligent Security Systems Research Laboratory, Division of Computer Science, University of Memphis, USA.

[8] J. W. Kim, Integrating Artificial Algorithms for Intrusion Detection, $\mathrm{PhD}$ thesis in Computer Science, University of London, 2002. 
[9] J. Mwakalinga, and L. Yngström, Sketch of a generic security framework based on the paradigms of SystemicHolistic Approach and the immune system, Proceedings of the Information Security South Africa, South Africa, 2005.

[10] Cheng, Y. A comprehensive Security Infrastructure for Mobile Agents, ISRN SU-KTH/DSV/R—97/13-SE, Stockholm, 1997.

[11] Yngström, L. A Systemic-Holistic Approach to academic programs in IT Security, $\mathrm{PhD}$ thesis, Stockholm University / Royal Inst. of Technology, Stockholm, Sweden, 1996.

[12] S. Beer, "The heart of the Enterprise," John Wiley \& Sons, London, 1979.

[13] Slay, J., Darzanos, K., Quirchmayr, G., \& Koronios, A. Towards a mature understanding of "culture" in information systems security research. Insights from Research. University of South Australia, School of Computer and Information Science, Mawson Lakes, Australia, 2003.

[14] N. Van Dam, V. Evers, and F. Arts, Cultural user experience, issues in e-government: Designing for a Multicultural society. Digital Cities 3, University van Amsterdam, Netherlands. 2003

[15] G. Miller, Living Systems, McGraw Hill, New York, 1978.
[16] G.H. Hofstede, Culture Consequences: International Differences in Work-related Values, Sage Productions, London, 2001.

[17] W. Cobern, "The Cultural Nature of the Concept Scientific Worldview," http://www.ouhk.edu.hk/ rcwww/misc/cobern.htm, Accessed 15 June, 2009.

[18] J. Slay, Human activity systems: A theoretical framework for designing learning for multicultural settings, Educational Technology \& Society 5 (1), 2002.

[19] S. Kowalski, IT Insecurity: a Multi-disciplinary Inquiry, $\mathrm{PhD}$ thesis, Royal Institute of Technology, Stockholm, Sweden, 1994.

[20] P. Brusilovsky, Knowledge Tree: A Distributed Architecture for Adaptive E-Learning, ACM 1-58113-9128/04/0005, 2004

[21] J. Mwakalinga, The holistic and immune security framework based on the principles of the Systemic-Holistic Approach and of the immune system, Ph D thesis, department of computer system sciences, Stockholm university / Royal Institute of technology, Sweden, 2009.

[22] ISO 27001 standard, http://27001.denialinfo.com/pdca.htm, 2008, Accessed 11 July, 2009. 